\title{
Importance of iron chelation therapy
}

\author{
A. Varoğlu \\ Northern Cyprus Thalassaemia Society
}

\begin{abstract}
It is necessary to remember that today patients have different options of chelation treatment, as desferrioxamine, deferiprone and deferasirox are available. However, a patient has to be compliant with treatments. They have always to remember that too much iron causes different complications and could be a barrier for a definitive cure from thalassemia.
\end{abstract}

\section{Iron chelation compliance and challenges}

Thalassaemia is an inherited blood disorder which in its most severe form causes life threatening anaemia.

The need for life long-blood transfusions results in iron overload in the body. Iron chelation agents are therefore necessary for the excretion of iron.

\section{Too much iron causes}

Endocrine Complications, Cardiac Complications, Organ damage (liver, endocrine glands and bones), Hepatic Complications and Infections (hepatitis $\mathrm{B}, \mathrm{C}$ and HIV)

The goal of chelation treatment is to prevent accumulation of harmful levels of body iron. Iron Chelation treatment should start when Serum Ferritin reaches about $1000 \mu \mathrm{g} / \mathrm{L}$.

The main issue for thalassaemia patients is conforming with or agreeing to do something, Compliance can be defined as the extent to which a patient's behaviour coincides with the doctor's prescription.

\section{Correspondence: A. Varoğlu, Northern Cyprus Thalassaemia Society.}

Key words: iron chelation therapy.

(C) Copyright A. Varoğlu, 2011

Licensee PAGEPress, Italy

Thalassemia Reports 2011; 1(s2):e11

doi:10.4081/thal.2011.s2.e11

This article is distributed under the terms of the Creative Commons Attribution Noncommercial License (by-nc 3.0) which permits any noncommercial use, distribution, and reproduction in any medium, provided the original author(s) and source are credited.

Parts of this work were presented at the "12th International Conference on Thalassemia and Hemoglobinopathies", Antalya (Turkey), 11-14 May 2011.
May compliance be a marker of quality of care? Answer is YES. Is non-compliance a problem? Answer is YES.

There are three iron chelators available now: desferrioxamine, deferiprone and deferasirox.

Desferrioxamine (DFO): available for more than 30 years. It has a short half life so must be given by continuous infusions, is administrated 8-12 hrs/day 5-7 days/week. Side effects involve the eyes, ears, bones, allergies, etc.).

Deferiprone (DFP): patented in 1982/licensed in EU 1999. It is taken 3 times/day orally. Side effects include vomiting, nausea, abdominal pain, joint pain, neutropenia, agranulocytosis).

Deferasirox (Exjade): is licensed in the US and the EU. It is given once daily by mouth. Side effects include nausea, skin rash, diarrhoea, vomiting, abdominal, pain, increase in serum creatinine levels.

\section{Barriers and Aids to compliance}

- Practical Aspects (uncomfortable and time consuming).

- Infusion Device (infusion pumps disadvantages): Restriction in activities (not sleeping properly, sports), Size of Pumps, Technical defects (dead battery, repair problems), Noise.

- Needle type causing discomfort.

- Local reactions.

\section{The doctor's part}

Doctor's and Nurses motivation should always be clear in seeking the optimum for their patients. Doctor's scientific evidence should always be clear cut in favour of the patient interest. Voluntary cooperation of the patient in taking drugs or medicine as prescribed must be the goal of the healthcare team through encouragement and motivation.

Thalassemia Patients are asked to comply with all or many of the following treatments: Blood Transfusion, Iron Chelation, HCV/Liver disease treatment.

Bone treatment, Diabetes, Cardiological monitoring, Fertility treatment, Endocrinological treatment, Psychosocial issues Specific other complications.

The burden to the patients life should be understood.

Although treatment has been getting better, patients are still non compliant Why?

- Acceptance the situation,

- pain,

- side effects,

- feeling different,

- body image damage, 
- getting bored,

- ignorance of parents,

- limitation of activities,

- lack of drugs!!

- cost of drugs (Exjade).

\section{Reason for missing desferal}

Needing a Break (10 days!), Too Tired, On Vacation today, Battery is dead, No good places for injection left, Feeling Fine these days.

\section{Compliance to treatment}

After DFP (and combination of DFP and Desferal) had been initiated as an iron chelator agent in 2000 , the rate of deaths as a result of cardiac diseases has dropped. Deferiprone monotherapy and combination therapy are effective as the following ways: decreasing serum ferritin, lowering liver iron overload, decreasing heart iron load (MRI T2*), improving cardiac functions, increasing the survival in cardiac disease.

EXJADE (ICL 670): Exjade monotherapy is effective in decreasing liver iron load and recently a possible study has also showed that Exjade is effective in reducing heart iron load.

DESFERAL: there is evidence for the effectiveness on long term survival and (for those who are fully compliant) in preventing complications of iron overload. 